\title{
Influence of collisions on ion dynamics in the inner comae of four comets
}

\author{
K. E. Mandt ${ }^{1}$, A. Eriksson ${ }^{2}$, A. Beth ${ }^{3}$, M. Galand ${ }^{3}$, and E. Vigren ${ }^{2}$ \\ ${ }^{1}$ Johns Hopkins University Applied Physics Laboratory, 11100 Johns Hopkins Rd., Laurel, MD 20723, USA \\ e-mail: Kathleen. Mandt@ jhuapl .edu \\ 2 Swedish Institute of Space Physics, PO Box 537, 751 21, Uppsala, Sweden \\ ${ }^{3}$ Department of Physics, Imperial College London, Prince Consort Road, London SW7 2AZ, UK
}

Received 11 December 2018 / Accepted 5 March 2019

\begin{abstract}
Context. Collisions between cometary neutrals in the inner coma of a comet and cometary ions that have been picked up into the solar wind flow and return to the coma lead to the formation of a broad inner boundary known as a collisionopause. This boundary is produced by a combination of charge transfer and chemical reactions, both of which are important at the location of the collisionopause boundary. Four spacecraft measured ion densities and velocities in the inner region of comets, exploring the part of the coma where an ion-neutral collisionopause boundary is expected to form.

Aims. The aims are to determine the dominant physics behind the formation of the ion-neutral collisionopause and to evaluate where this boundary has been observed by spacecraft.

Methods. We evaluated observations from three spacecraft at four different comets to determine if a collisionopause boundary was observed based on the reported ion velocities. We compared the measured location of the ion-neutral collisionopause with measurements of the collision cross sections to evaluate whether chemistry or charge exchange are more important at the location where the collisionopause is observed.

Results. Based on measurements of the cross sections for charge transfer and for chemical reactions, the boundary observed by Rosetta appears to be the location where chemistry becomes the more probable result of a collision between $\mathrm{H}_{2} \mathrm{O}_{\text {and }} \mathrm{H}_{2} \mathrm{O}^{+}$than charge exchange. Comparisons with ion observations made by Deep Space 1 at 19P/Borrelly and Giotto at 1P/Halley and 26P/Grigg-Skjellerup show that similar boundaries were observed at 19P/Borrelly and 1P/Halley. The ion composition measurements made by Giotto at Halley confirm that chemistry becomes more important inside of this boundary and that electron-ion dissociative recombination is a driver for the reported ion pileup boundary.
\end{abstract}

Key words. molecular processes - plasmas - comets: individual: 67P/Churyumov-Gerasimenko - comets: individual: 1P/Halley comets: individual: 19P/Borelly - comets: individual: 26P/Grigg-Skjellerup

\section{Introduction}

Several cometary flyby missions have provided information about the interaction of the solar wind with a coma. Some of the more interesting features in these observations include detection and characterization of plasma interaction boundaries that demonstrate measurable changes in plasma parameters between one interaction region and the next (see Mandt et al. 2016, for a detailed review of boundary observations and theory). Plasma interaction boundaries can be permanent features, solar wind and interplanetary magnetic field (IMF) boundaries, or small-scale transient features created by instabilities or waves (Cravens 1989). The bow shock, a permanent boundary where mass loading of the solar wind flow causes it to transition from supersonic to subsonic, was observed by several spacecraft flybys (e.g., Galeev et al. 1986). Rosetta observed the bow shock of 67P/Churyumov-Gerasimenko (67P) shortly after it first formed (Gunell et al. 2018), which is a stage of development not previously observed by any mission. However, only the Rosetta mission (Goetz et al. 2016, 2017; Nemeth et al. 2016) and the Giotto flyby of Halley (Neubauer et al. 1986) approached close enough to the nucleus to detect the boundary of the diamagnetic cavity, a magnetic-field free region surrounding the comet nucleus.
Rosetta made several observations of a diamagnetic cavity boundary, and work is ongoing to better understand this feature.

Several boundaries have been observed between the bow shock and the diamagnetic cavity. One of these is the cometopause, where the composition of the ions flowing in the antisunward direction as part of the solar wind flow changes from predominantly solar wind ions to predominantly cometary ions (Gringauz et al. 1986a; Gombosi 1987; Fuselier et al. 1988; Mendis et al. 1989; Coates 1997). Cravens (1989) defined the cometopause as the collisionopause for solar wind proton charge exchange. The location and width of this boundary vary with time as a result of changes in the solar wind and the neutral gas flow parameters (Gringauz \& Verigin 1991).

Before the Rosetta mission (Glassmeier et al. 2007; Taylor et al. 2017), exploration of the region between the cometopause and the diamagnetic cavity boundary had been limited. Because Rosetta rendezvoused with $67 \mathrm{P}$ and escorted the comet for an extended portion of its orbit around the Sun, the observations allowed detailed exploration of the inner boundaries of the comet-solar wind interaction as they formed and evolved (Mandt et al. 2016; Behar et al. 2017; Nilsson et al. 2017; Timar et al. 2017; Odelstad et al. 2018). Between the cometopause and the diamagnetic cavity boundary, the dominant ions in the solar 


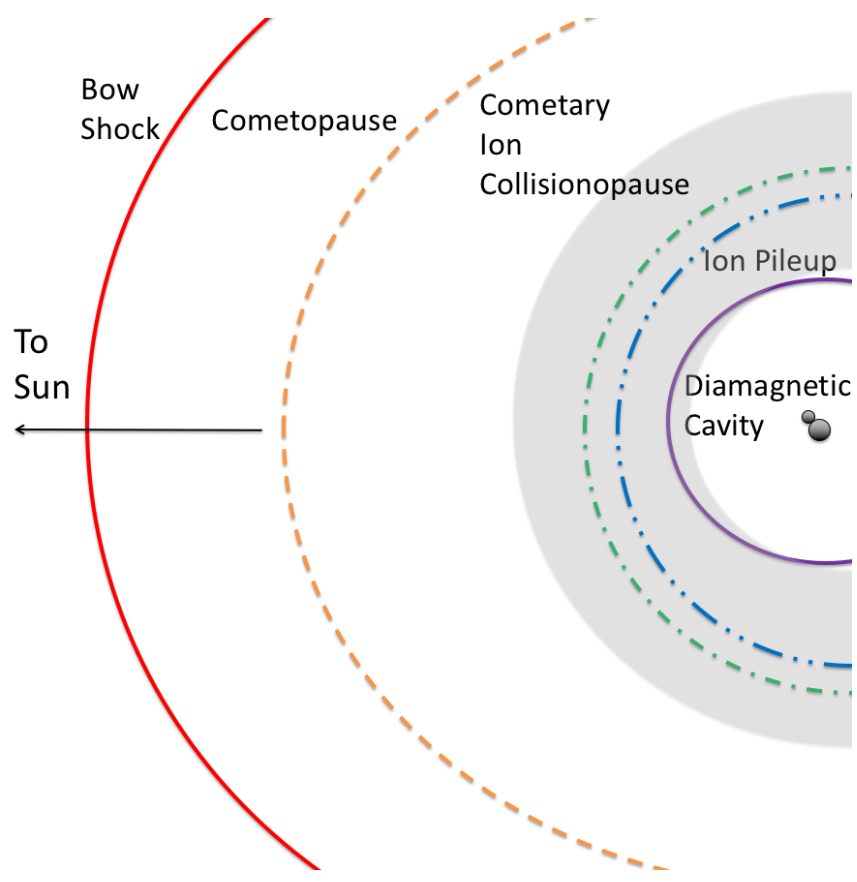

Fig. 1. Illustration of the plasma interaction boundaries that have been observed at more than one of the comets listed in Table 1 (not to scale). The boundaries are as follows: bow shock (red solid line), cometopause (orange dashed line), cometary ion collisionopause (green dash-dot line), ion pileup (blue dash-dash-dotted line), and diamagnetic cavity boundary (purple solid line). Observed distances where applicable are listed in Table 1 for four comets. The gray shaded region is the portion of the comet solar wind interaction explored by Rosetta between May and December 2015. This is a schematic and does not accurately represent the shape of the boundaries. For instance, the diamagnetic cavity boundary seems to be more structured than this simplified view shown here in violet (e.g., Henri et al. 2017).

wind flow are picked up cometary ions (Mendis et al. 1989). In this region, the Vega probes (Gringauz et al. 1986b; Vaisberg et al. 1987) and Giotto observed a region where ion densities increased with decreasing distance to the comet, termed the ion pileup region (Balsiger et al. 1986). $\mathrm{H}_{3} \mathrm{O}^{+}$was found by Giotto to be the dominant ion species in the ion pileup region (Balsiger et al. 1986; Schwenn et al. 1988). An ion pileup boundary located inward of the pileup region was reported, where the ion density dropped by a factor of 4 similar in the comet ward direction and the ion density profile changed from $1 / r^{2}$ to $1 / r$ (Pätzold et al. 1997; Coates 1997, 2009), which was proposed to form as a result of decreasing electron temperature approaching the nucleus, leading to increasing electron recombination rates with decreasing distance from the comet (Ip et al. 1988; Gan \& Cravens 1990; Häberli et al. 1995, 1996; Lindgren et al. 1997). Unfortunately, this interpretation has not been confirmed by Giotto observations because spacecraft charging effects did not allow measurement of the temperature of the thermal electrons relevant to the recombination rate of ions in the pileup region (Häberli et al. 1996). A schematic summarizing the different regions and boundaries in given as illustrated in Fig. 1.

Mandt et al. (2016) reported observations by Rosetta of the formation of a boundary between two interaction regions characterized by ions with high energies outside of the boundary and stagnant with low energies inside. The latter were detected only because the spacecraft was negatively charged. They were measured using the Rosetta Plasma Consortium (RPC, Carr et al. 2007) Ion Electron Spectrometer (RPC-IES, Burch et al. 2007), which consists of two electrostatic analyzers that measure ions and electrons with energies per charge between $4.3 \mathrm{eV} \mathrm{q}^{-1}$ and $18 \mathrm{keV} \mathrm{q}^{-1}$. Because the high-energy ions are observed to be flowing toward the nucleus in the antisunward direction Goldstein et al. (2017), they were interpreted to be cometary ions picked up by the solar wind at a distance and brought back toward the nucleus as part of the mass-loaded solar wind flow. The lowenergy ions inside of the boundary are predominantly made up of recently ionized ions that are detected at energies similar to the magnitude of the negative charge on the spacecraft. The newborn ions inside of the boundary begin with the same energy as the neutrals, $\sim 0.1 \mathrm{eV}$ (Vigren et al. 2015), but could be accelerated by an ambipolar electric field to energies up to $10 \mathrm{eV}$ (Vigren et al. 2017). However, the energy resolution of RPC-IES is too poor at low energies to differentiate between the newborn ions and ions that have been accelerated by the ambipolar electric field. Therefore, the apparent energy of the ions inside of the boundary is dominated by the influence of the spacecraft charge. We interpret the boundary observed by RPC-IES to be a collisionopause formed between the picked up cometary ions flowing with the mass loaded solar wind and the cometary neutrals. The clearest observations of the collisionopause were made during an excursion to $1500 \mathrm{~km}$ distance from the comet, which took place during September and October 2015. These observations demonstrated that the collisionopause existed for longer than any transient feature of the solar wind and was thus a permanent feature of the comet-solar wind interaction.

In order to test whether other comet missions have observed a cometary ion collisionopause and to evaluate which physical parameters determine the distance of this boundary from the nucleus, we compare the ion density and velocity observed by Rosetta during the excursion with similar measurements reported for three other comet flybys. The ion density and velocity are determined by calculating the ion moments measured by RPC-IES after first correcting for spacecraft charging effects. In the environment of the coma, the spacecraft charge is typically negative and causes an increase in the observed ion energy of the same magnitude as the spacecraft charge (e.g., a $-10 \mathrm{~V}$ charge increases all ion energy by $10 \mathrm{eV}$ ). We use the spacecraft charge (Odelstad et al. 2015, 2017) measured by the RPC Langmuir Probe (RPC-LAP, Eriksson et al. 2007) to correct ion energies and then derive the velocity moment. We use electron densities derived by RPC-LAP as a proxy for the ion densities because the limited field of view of RPC-IES makes density determinations unreliable. Observations from spacecraft flybys used for this study are compared to those at 67P by Rosetta in Table 1.

\section{Collisionopause boundaries}

A collisionopause is a boundary where collisions first become important inside of the boundary (Mendis et al. 1986; Cravens 1989, 1991). Cravens (1991) outlined various types of collisionopause for both ions and electrons depending on the collision processes. We outline in Table 2 the ion interactions that could cause collisionopause boundaries and give example reactions that could take place as a result of an ion collision. For the evaluation of the RPC-IES-observed boundary, we focus on ion-neutral charge transfer (R1) and ion-neutral chemistry (R2).

On the one hand, charge transfer, reaction (R1), transfers the charge from fast cometary ions in the mass-loaded solar wind flow to a neutral traveling away from the comet at velocities of $\sim 1 \mathrm{~km} \mathrm{~s}^{-1}$ or lower. The ion becomes an energetic neutral, leading to a reduction in the number of high-energy ions relative to low-energy ions. Chemistry, on the other hand, changes the relative composition of the ion population in addition to the bulk 
Table 1. Comparison of Rosetta observations at comet 67P during the 2015 Rosetta excursion with flyby conditions and plasma boundaries detected at three comets: 19P/Borrelly by Deep Space 1, comet 1P/Halley and 26P/Grigg-Skjellerup (26P/GS) by Giotto.

\begin{tabular}{lcccc}
\hline \hline & 19P/Borrelly & $1 \mathrm{P} /$ Halley & 26P/GS & $67 \mathrm{P}$ \\
\hline Date of observation (DD/MM/YYYY) & $22 / 09 / 2001$ & $14 / 03 / 1986$ & $10 / 07 / 1992$ & $22 / 09-11 / 10 / 2015$ \\
Production rate $\left(\mathrm{s}^{-1}\right)$ & $3.5 \times 10^{28}$ & $3.8 \times 10^{29}$ & $7 \times 10^{27}$ & $\sim 10^{28}$ \\
Heliocentric distance $(\mathrm{au})$ & 1.36 & 0.903 & 1.01 & 1.4 \\
Solar wind speed $\left(\mathrm{km} \mathrm{s}^{-1}\right)$ & 360 & $350-400$ & 360 & Variable \\
Closest approach $(\mathrm{km})$ & 2171 & 605 & 200 & $300-1500$ \\
Spacecraft velocity $\left(\mathrm{km} \mathrm{s}^{-1}\right)$ & 16.5 & 68.4 & 13.99 & $<0.002$ \\
Bow shock $(\mathrm{km})$ & $1.52 \times 10^{5(a)}$ & $5.98 \times 10^{5(b)}$ & $1.67 \times 10^{4(c)}$ & Detected as it formed ${ }^{(f)}$ \\
Cometopause $(\mathrm{km})$ & $1.51 \times 10^{4(a)}$ & $1.4 \times 10^{5(d)}$ & $1.8 \times 10^{3(e)}$ & $\boldsymbol{X}^{(h)}$ \\
Cometary ion collisionopause $(\mathrm{km})$ & $3.5 \times 10^{5}$ & $2.7 \times 10^{4(g)}$ & $\boldsymbol{X}$ & $500-700^{(h)}$ \\
Ion pile-up boundary $(\mathrm{km})$ & $\boldsymbol{X}$ & $10^{4(g)}$ & $\boldsymbol{X}$ & $\boldsymbol{X}^{(j)}$ \\
Diamagnetic cavity $(\mathrm{km})$ & $\boldsymbol{X}$ & $4.6 \times 10^{3(b, i)}$ & $\boldsymbol{X}$ & $\sim 170^{(j)}$ \\
\hline
\end{tabular}

Notes. The ion pileup region, which is the focus of this study, is located between the ion velocity drop designated as the cometary ion collisionopause and the ion pileup boundary. Crosses mean that the corresponding plasma boundary has not been observed.

References. ${ }^{(a)}$ Young et al. (2004). ${ }^{(b)}$ Coates (2009). ${ }^{(c)}$ Coates et al. (1996). ${ }^{(d)}$ Rème et al. (1988). ${ }^{(e)}$ Johnstone et al. $(1993) .{ }^{(f)} \mathrm{Gunell} \mathrm{et} \mathrm{al.}(2018)$. ${ }^{(g)}$ Schwenn et al. (1988). ${ }^{(h)}$ Mandt et al. (2016). ${ }^{(i)}$ Neubauer (1988). ${ }^{(j)}$ Not observed during excursion, but observations reported by Goetz et al. (2016, 2017).

Table 2. Reactions that result from collisions between ions, neutrals, and electrons in a coma.

\begin{tabular}{|c|c|c|c|c|c|c|c|c|c|c|}
\hline Number & Type & Example & & & & & & & & \\
\hline (R1) & Ion-neutral charge transfer & $\mathrm{H}_{2} \mathrm{O}_{\text {fast }}^{+}$ & + & $\mathrm{H}_{2} \mathrm{O}$ & $\longrightarrow$ & $\mathrm{H}_{2} \mathrm{O}_{\text {fast }}$ & + & $\mathrm{H}_{2} \mathrm{O}^{+}$ & & \\
\hline (R2) & Ion-neutral chemistry & $\mathrm{H}_{2} \mathrm{O}^{+}$ & + & $\mathrm{H}_{2} \mathrm{O}$ & $\longrightarrow$ & $\mathrm{H}_{3} \mathrm{O}^{+}$ & + & $\mathrm{HO}$ & & \\
\hline (R3) & Ionization & $\mathrm{H}_{\text {fast }}^{+}$ & + & $\mathrm{H}_{2} \mathrm{O}$ & $\longrightarrow$ & $\mathrm{H}_{\text {fast }}^{+}$ & + & $\mathrm{H}_{2} \mathrm{O}^{+}$ & + & $e^{-}$ \\
\hline (R4) & Electron removal & $\mathrm{O}^{\text {fas }}$ & + & $\mathrm{H}_{2} \mathrm{O}$ & $\longrightarrow$ & $\mathrm{O}^{+}$ & + & $\mathrm{H}_{2} \mathrm{O}$ & + & $e^{-}$ \\
\hline (R5) & Electron-ion dissociation recombination & $\mathrm{H}_{3} \mathrm{O}^{+}$ & + & $e^{-}$ & $\longrightarrow$ & $\mathrm{HO}$ & + & $2 \mathrm{H}$ & & \\
\hline (R6) & Ion-ion Coulomb interaction & $\mathrm{H}_{2} \mathrm{O}^{+}$ & + & $\mathrm{H}_{2} \mathrm{O}^{+}$ & $\longrightarrow$ & $\mathrm{H}_{2} \mathrm{O}^{+}$ & + & $\mathrm{H}_{2} \mathrm{O}^{+}$ & & \\
\hline
\end{tabular}

Notes. These reactions are related to different types of collisionopause boundaries (Cravens 1991).

energy of the ions. For example, in reaction ( $\mathrm{R} 2)$, a proton is transferred from $\mathrm{H}_{2} \mathrm{O}^{+}$to $\mathrm{H}_{2} \mathrm{O}$, creating an $\mathrm{H}_{3} \mathrm{O}^{+}$ion and changing the bulk mass $19 / 18$ ratio of the ion population. If $\mathrm{H}_{2} \mathrm{O}^{+}$is fast, it will end as an energetic neutral, while $\mathrm{H}_{3} \mathrm{O}^{+}$will have the same energy as the neutrals. Another reaction that should be noted is the proton transfer from $\mathrm{H}_{3} \mathrm{O}^{+}$to $\mathrm{NH}_{3}$, producing $\mathrm{NH}_{4}^{+}$ in the dense coma (Beth et al. 2016). This reaction depends on the volume mixing ratio of $\mathrm{NH}_{3}$ in the coma and will reduce the mass $19 / 18$ ratio.

As stated earlier, the cometopause is suggested to be the location where ion-neutral charge transfer of the solar wind ions with the cometary neutrals becomes dominant. It corresponds to a region where ion energy and composition would change (Cravens 1991). The relevant reaction for the cometopause is $\mathrm{H}_{\text {fast }}^{+}+\mathrm{H}_{2} \mathrm{O} \longrightarrow \mathrm{H}_{\text {fast }}+\mathrm{H}_{2} \mathrm{O}^{+}$, which is similar to (R1) in Table 2. In this region, $\mathrm{H}^{+}$becomes energetic hydrogen and a slow $\mathrm{H}_{2} \mathrm{O}^{+}$is produced. The bulk composition of the ions changes from solar wind protons to cometary ions (primarily $\mathrm{H}_{2} \mathrm{O}^{+}$), and the ion bulk energy is reduced.

The boundary that we propose to identify with Rosetta observations is where both (R1) and (R2) are acting to reduce the bulk ion energy and are changing the cometary ion composition, such that $\mathrm{H}_{3} \mathrm{O}^{+}$is the dominant ion inward of the boundary.

\subsection{Ion-neutral collisionopause}

Observations with Rosetta demonstrated the importance of ion-neutral and electron-neutral collisionopause boundaries (Mandt et al. 2016; Eriksson et al. 2017; Henri et al. 2017;
Engelhardt et al. 2018). Many studies have treated the location of the ion-neutral collisionopause (Mendis et al. 1986, 1989; Mandt et al. 2016; Galand et al. 2016) as an ion exobase. This would be similar to the boundary used in aeronomy, where collisions dominate the dynamics of the gas inside of the boundary. The location of an exobase is where the Knudsen number, or the ratio of the mean free path to the scale height, is equal to one. In a comet, the plasma mean free path, $\lambda$, is given by

$\lambda=\frac{1}{n_{\mathrm{n}} \sigma_{\mathrm{C}}}$,

where $\sigma_{\mathrm{C}}$ is the ion-neutral collision cross section and $n_{\mathrm{n}}$ is the neutral number density. This can also be used for electrons to determine the electron-neutral collisionopause location, or electron exobase (e.g., Mandt et al. 2016; Henri et al. 2017). The ion-neutral collision cross section was estimated to be between $2 \times 10^{-15} \mathrm{~cm}^{2}$ for solar wind ions and $8 \times 10^{-15} \mathrm{~cm}^{2}$ for massloaded solar wind with a bulk composition of $\mathrm{H}_{2} \mathrm{O}^{+}$(Mendis et al. 1986), while the electron-neutral collision cross section for $5 \mathrm{eV}$ electrons is $5 \times 10^{-16} \mathrm{~cm}^{2}$ (Itikawa \& Mason 2005).

Several Rosetta studies have concluded that a $1 / r$ plasma density profile is a good description of the plasma density with distance from the comet. Using data from two close nucleus flybys obtained at 2.35 AU, Edberg et al. (2015) found this to describe the data well. Heritier et al. (2017) obtained a similar result at the end-of-mission descent of Rosetta to the nucleus at 3.8 AU, down to a few nucleus radii when the plasma density was found to decrease toward the nucleus, as expected. 
Furthermore, Galand et al. (2016), Vigren et al. (2016), and Heritier et al. $(2017,2018)$ have shown through a Rosetta multiinstrument study that the electron density at large heliocentric distances is well described by a $1 / r$ model, assuming that the ion velocity is the same as the neutrals, in agreement with low outgassing activity (Beth et al. 2019). Near perihelion, the theory still predicts a $1 / r$ dependence with some assumptions (e.g., constant ion velocity and electron temperature) in the region probed by Rosetta. Although the model overestimates the observed electron number density during this period, it does not preclude a decrease as $1 / r$ in the inner coma. The magnitude can be higher while the scale height remains barely changed (Beth et al. 2019). This is what is observed. Therefore, we assume that this scale height is effective for the entire mission, and can find the location of the collisionopause by setting the mean free path equal to the distance from the comet,

$\lambda=r=\frac{\sigma_{\mathrm{C}} Q}{4 \pi u_{\mathrm{n}}}$,

where $Q$ is the production rate in per second, and $u_{\mathrm{n}}$ is the velocity of the neutrals in $\mathrm{m} \mathrm{s}^{-1}$. This is obtained by substituting in Eq. (1) the neutral density with the relation valid for a gas expanding at a constant bulk velocity,

$n_{\mathrm{n}}(r)=\frac{Q}{4 \pi u_{\mathrm{n}} r^{2}}$.

A different approach was proposed by Cravens (1991) for determining the location of an ion-neutral collisionopause in a coma. This method defines the collisionopause as the location where the transport time is equal to the collision time. For comets, the ion transport time scale, $\tau_{\mathrm{T}}$, is given as

$\tau_{\mathrm{T}}=\frac{r}{u_{\mathrm{i}}}$,

where $r$ is the cometocentric distance and $u_{\mathrm{i}}$ is the ion bulk velocity. The collision timescale depends on the type of interaction. In the case of an ion-neutral collision process designated as $p$, this is given as

$\tau_{\mathrm{C}}=\frac{1}{u \sigma_{\mathrm{p}} n_{\mathrm{n}}}$

where $u$ is the relative velocity between the ions and the neutrals and $\sigma_{\mathrm{p}}$ is the cross section for the process $\mathrm{p}$. Setting the transport timescale equal to the collision timescale, substituting Eq. (3) for $n_{\mathrm{n}}$ and solving for $r$ gives an estimate for the location of the collisionopause for process $\mathrm{p}$

$r=\frac{u \sigma_{\mathrm{p}} Q}{4 \pi u_{\mathrm{n}} u_{\mathrm{i}}}$.

We note that Eq. (2) is similar to Eq. (6), except that using the exobase approach given in Eq. (2) requires that the velocity of the ions relative to the neutrals, $u$, is approximately equal to the ion velocity, $u_{\mathrm{i}}$, such that $u / u_{\mathrm{i}} \approx 1$. This is a valid assumption when the ion velocity is $\sim 400 \mathrm{~km} \mathrm{~s}^{-1}$, as is common for the solar wind. This may not be valid deep within the coma, however, where the ion flow velocity is significantly reduced. For example, if the ion velocity is $10 \mathrm{~km} \mathrm{~s}^{-1}$ and is flowing opposite the comet neutral velocity of $1 \mathrm{~km} \mathrm{~s}^{-1}$, the relative velocity is $11 \mathrm{~km} \mathrm{~s}^{-1}$ and the distance of the collisionopause from the nucleus is multiplied by $11 / 10$, increasing the distance by $10 \%$. However, it is important to note that the relative velocity of ions with regard to the neutrals can be accounted for in Eq. (2) by recognizing the dependence of the collision cross section on the energy of the collision. We review laboratory and theoretical studies of relevant ion-neutral collision cross sections in Sect. 2.2.

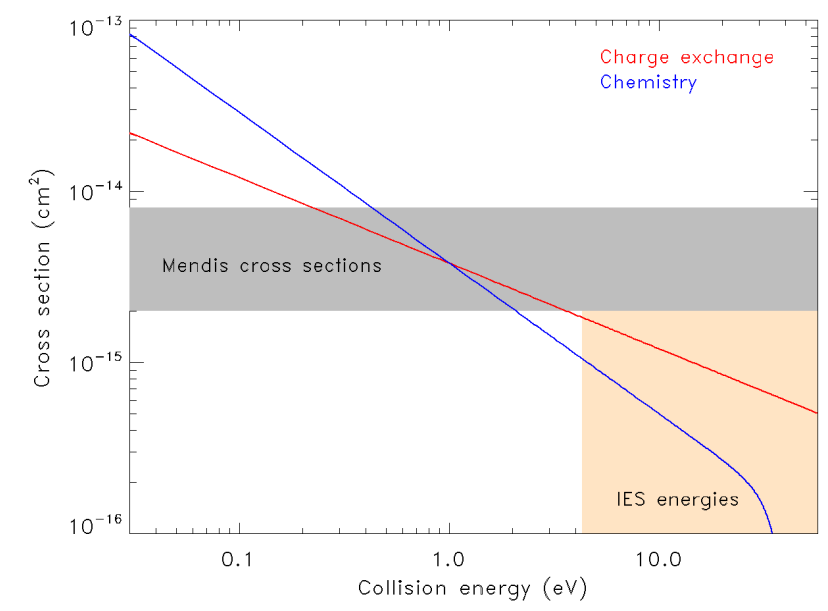

Fig. 2. Comparison of the Fleshman et al. (2012) cross-section fits to the $\mathrm{H}_{2} \mathrm{O}^{+}+\mathrm{H}_{2} \mathrm{O}$ collision cross section measurements by Lishawa et al. (1990) for reactions ( $\mathrm{R} 1$; red line) and (R2; blue line) with the crosssection values used by Mendis et al. (1986; shaded gray area) as a function of the relative energy of the reacting pair. The ion energy range sampled by RPC-IES is given by the tan box (may be shifted toward lower energy for a negative $\mathrm{S} / \mathrm{C}$ potential).

\subsection{Ion-neutral cross sections and the collisionopause distance}

One of the greatest sources of uncertainty in calculating the location of the collisionopause is the collision cross section. As stated in Sect. 2.1, the values that have typically been used for the ion-neutral collision cross-sections in a coma are given as $2 \times 10^{-15} \mathrm{~cm}^{-2}$ for solar wind ions and $8 \times 10^{-15} \mathrm{~cm}^{2}$ for massloaded solar wind with a bulk composition of $\mathrm{H}_{2} \mathrm{O}^{+}$in Mendis et al. (1986). However, according to Johnson et al. (2008), collision cross sections depend on the composition of the ions and neutrals as well as on the energy of the ions.

Lishawa et al. (1990) measured the collision cross section for charge exchange between $\mathrm{H}_{2} \mathrm{O}^{+}$and $\mathrm{H}_{2} \mathrm{O}$ (R1 in Table 2) and for the bimolecular reaction to produce $\mathrm{H}_{3} \mathrm{O}^{+}$(R2 in Table 2) over collision energies between 0.5 and $25 \mathrm{eV}$. They found that the collision cross section decreased as the relative energy of the reacting pair increased and that the rate of decrease with energy was much higher for bimolecular reactions than for charge exchange. This is illustrated in Fig. 2, where the fits from Fleshman et al. (2012) to the Lishawa et al. (1990) measurements are shown over a range of energies relevant to the inner region of the coma. The cross sections for both reactions are equal within the range of values recommended by Mendis et al. (1986; gray shaded area in Fig. 2).

Mandt et al. (2016) used observations of ions with energies between 4 and $1000 \mathrm{eV}$ made by the Rosetta Plasma Consortium Ion Electron Spectrometer (RPC-IES, Burch et al. 2007) to identify locations where Rosetta crossed the ion-neutral collisionopause boundary. We identified the boundary location as the point where the pickup-ion energies dropped to energies below the RPC-IES lower limit of $4.32 \mathrm{eV}$ if the spacecraft potential was greater than $-4 \mathrm{~V}$ or to the spacecraft potential value if the spacecraft potential was less than $-4 \mathrm{~V}$ (Odelstad et al. 2015) as measured by the RPC-LAP. Therefore, these crossings were defined based on the instrument detection limits rather than comparison between ion and neutral energies. The energy range of the ions that RPC-IES was capable of observing and the respective ion-collision cross sections are shown in the tan shaded region of Fig. 2. The ion energy observed by RPC-IES is the original ion energy plus any acceleration imparted by the $\mathrm{S} / \mathrm{C}$ 


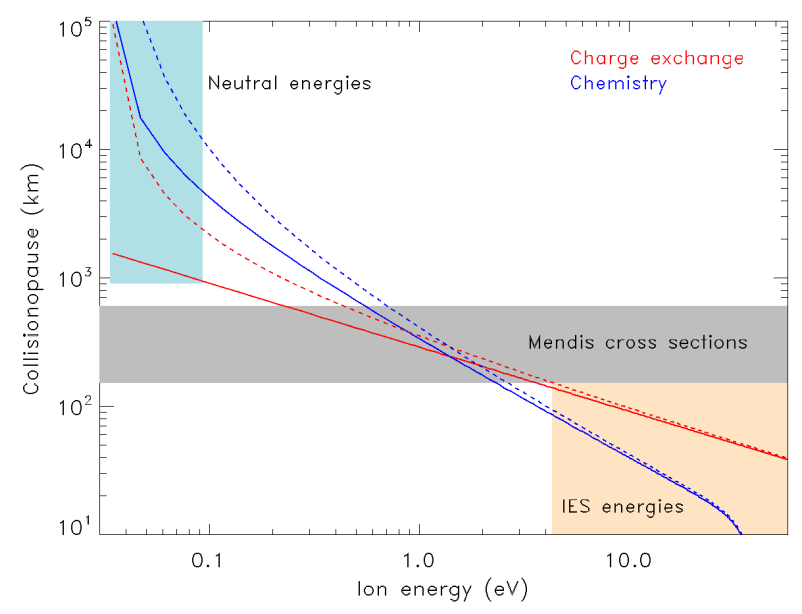

Fig. 3. Location of the collisionopause assuming an outgassing rate of $6 \times 10^{27} \mathrm{~s}^{-1}$, the value estimated for $67 \mathrm{P}$ during the Rosetta excursion. The dashed lines give the location using Eq. (2), while the solid lines include the ion velocity correction given in Eq. (6). A neutral outgassing velocity of $600 \mathrm{~m} \mathrm{~s}^{-1}$ is assumed. The collisionopause location was observed by RPC-IES at 500-700 km, or the upper range using the Mendis cross sections (gray band). The blue shaded region indicates the location where the ion and neutral velocities are the same. The tan box indicates the range of IES ion energy, but this range is extended toward lower energies because of the negative spacecraft potential.

potential, so that ions with energies below the instrument lower limit of $4 \mathrm{eV}$ were observed at values equal to the magnitude of the negative charge on the spacecraft.

The ions are considered fully collisional when their energy is the same as the neutral energies, which would be the value at a collision energy close to zero. As Fig. 2 shows, the collision cross section increases exponentially as the collision energy approaches zero. The cross sections given by Mendis et al. (1986) are higher than the RPC-IES energy range when ignoring spacecraft charging effects, but are well below the energy range where the ions have the same velocity as the neutrals. This range of values includes the crossover point where (R1) is equally effective as (R2). This crossover point means that when collision energies are below $\sim 1 \mathrm{eV}$, collisions are more likely to result in chemical reactions than charge exchange, while the latter is more probable at energies above $\sim 1 \mathrm{eV}$.

In Fig. 3 we illustrate the location of the collisionopause assuming an outgassing rate relevant to the Rosetta excursion of $6 \times 10^{27} \mathrm{~s}^{-1}$ (Mandt et al. 2016). We show the collisionopause distance as a function of the ion energy using the exobase method from Eq. (2) (dashed lines) and from Eq. (6), which includes a correction for ion velocity (solid lines). The range of values for which the ion energy is in the same range as the neutral energies is illustrated in the blue shaded region.

Although ion velocity relative to the neutral velocity plays an important role in determining the location of the collisionopause in the neutral energy range, the cross sections relevant to the neutral energies does not predict the boundary locations observed by Rosetta during the excursion, which were at distances between 500 and $700 \mathrm{~km}$ from the nucleus (Mandt et al. 2016). We note that the crossings observed were in the upper range predicted by the Mendis cross sections in Fig. 3, where the collision energy is $0.2-1.0 \mathrm{eV}$ and chemistry becomes the dominant reaction resulting from collisions. This suggests that the boundary observed by Rosetta coincides with the region where chemistry becomes more important than charge exchange.

Examples of RPC-IES observations of ion-neutral collisionopause boundary crossings from the Rosetta excursion are

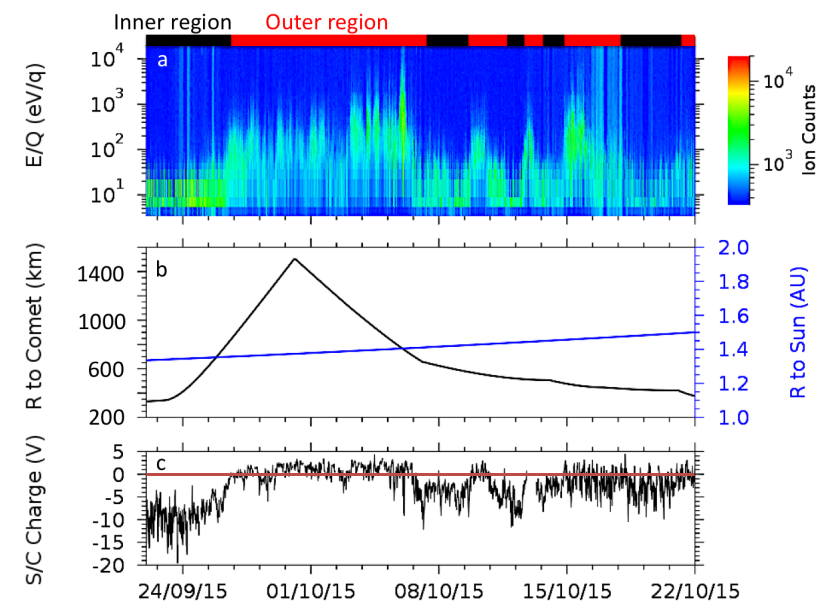

Fig. 4. Observations of the ion-neutral collisionopause crossings that took place in September and October 2015 during the excursion to $1500 \mathrm{~km}$. The bar at the top of the figure indicates where the spacecraft crosses from the inner (black) to the outer (red) region and back again. The inner region is where the ion energies (panel a) are observed at the spacecraft potential (panel c), which varied between 0 and $-20 \mathrm{~V}$. Ions with energies greater than $50 \mathrm{eV}$ are observed in the outer region. Some crossings appear to show a sharp boundary, such as the one on 15 October, while other crossings appear to represent a broad boundary. The boundary crossings that appear sharp likely do so because the solar wind dynamic pressure increased and caused the boundary to cross the spacecraft and not because the boundary itself is a sharp transition (see Mandt et al. 2016, for details).

illustrated in Fig. 4, where the transition between the inner and outer region at times appeared to be sharp and at other times appeared to be characterized by a gradual decrease in energy with decreasing distance from the boundary. Mandt et al. (2016) concluded that the boundary was wide and represented a gradual transition between collisional and non-collisional regions. We determined that boundary crossings likely appeared to be sharp as a result of changes in the solar wind dynamic pressure that forced the boundary to move past the spacecraft, which was moving at a rate much slower than the motion of the boundary in response to changes in the solar wind. As the observations illustrated in Fig. 4 show, when the spacecraft is in the outer region, the ion energy decreases as the spacecraft approaches the boundary and increases as the spacecraft moves away from the boundary.

\section{Observations during spacecraft flybys of comets}

Three other spacecraft flybys, described in Table 1, reported ion velocity and density as a function of distance from the nucleus during their flybys. We compare the Rosetta observations during the excursion with these flybys to better characterize the ion-neutral collisionopause in a coma. There are notable differences between the Rosetta mission and these three comet flybys, including the time spent near the comet and the spacecraft velocity. Rosetta moved at only a few meters per second relative to the nucleus, while the other spacecraft flew through the coma at very high velocities (tens of $\mathrm{km} \mathrm{s}^{-1}$ ). Furthermore, most of the flybys occurred with comets experiencing outgassing rates higher than the maximum observed with Rosetta (Hansen et al. 2016). The exception is the Giotto flyby of 26P/Grigg-Skjellerup (26P/GS), which had a relatively weak outgassing rate that was similar in magnitude to the maximum outgassing rate of $67 \mathrm{P}$ (see Table 1).

We illustrate in Fig. 5 relevant parameters for each of the comet observations as a function of distance from the nucleus 


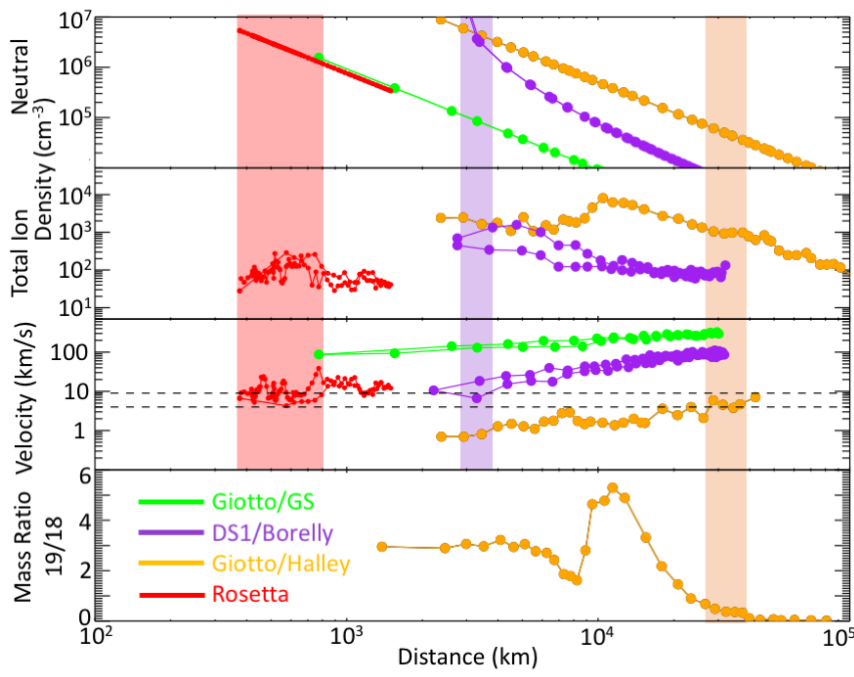

Fig. 5. Comparison of the neutral and plasma observations as a function of the distance from the nucleus as observed by Rosetta and the three comet flybys (see Table 1 for references for each dataset). Top panel: neutral number density determined based on the outgassing rate and Eq. (3). Second panel: total ion number density for the flybys and electron density for Rosetta. Third panel: ion bulk velocity. Bottom panel: ion ratio between mass $19\left(\mathrm{H}_{3} \mathrm{O}^{+}\right)$and mass 18 (mostly $\mathrm{H}_{2} \mathrm{O}^{+}$with some contribution from $\mathrm{NH}_{4}^{+}$). The horizontal dashed lines represent the estimated bulk velocities for RPC-IES observations of collisionopause crossings after correcting for spacecraft charging effects. The shaded regions indicate where Rosetta, DS1, and Giotto at Halley crossed into a similar range of velocities. The ion velocities at $26 \mathrm{P} / \mathrm{GS}$ were above this range during the entire flyby.

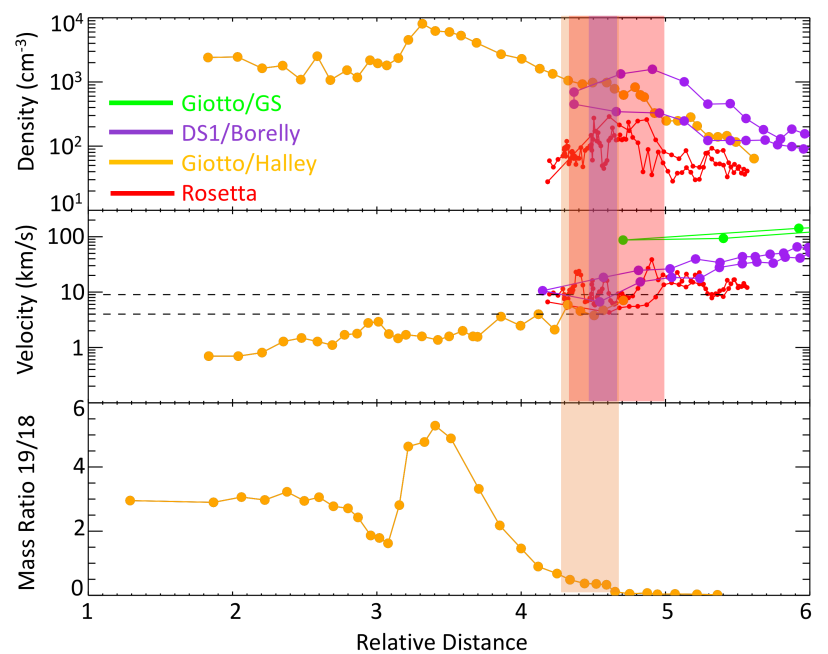

Fig. 6. Comparison of the same plasma observations from Fig. 5 with the distance scaled by the production rate using Eq. (7). See the caption of Fig. 5 for details.

and in Fig. 6 as a function of the distance scaled by the production rate. We scale the distance by production according to

$D_{\text {rel }}=\frac{10^{27} r}{Q}$

where $Q$ in $\mathrm{s}^{-1}$ and $r$ in $\mathrm{km}$ are multiplied by $10^{27} \mathrm{~s}^{-1}$ to give relative distance, $D_{\text {rel }}$, in $\mathrm{km}$.

The neutral densities in Fig. 5 are based on the production rate of each comet, while the ion densities and velocities are derived from the published ion moments for each flyby (Altwegg et al. 1993; Goldstein et al. 1994; Young et al. 2004). The
RPC-IES ion densities underestimate the total ion density because of the limited energy range and field of view of the instrument. Therefore, for Rosetta we provide the electron densities derived by RPC-LAP assuming charge neutrality (Mandt et al. 2016). The Giotto flyby of Halley (Altwegg et al. 1993) includes measurements of the mass 18 and 19 ions that can be used to estimate the efficiency of reaction (R2) in the coma based on the $\mathrm{H}_{3} \mathrm{O}^{+} / \mathrm{H}_{2} \mathrm{O}^{+}$ratio (M19/M18). No similar measurements are available for the other flybys or for Rosetta because the Rosetta mass spectrometer was shut off during the excursion. When M19/M18 is above one, the production of $\mathrm{H}_{3} \mathrm{O}^{+}$through reaction $(\mathrm{R} 2)$ is more efficient than the local ionization rate of $\mathrm{H}_{2} \mathrm{O}$. However, this ratio is also influenced by differences in the loss rates of $\mathrm{H}_{3} \mathrm{O}^{+}$and $\mathrm{H}_{2} \mathrm{O}^{+}$as discussed below, as well as by the production of $\mathrm{NH}_{4}^{+}$(Beth et al. 2016).

The panels in Fig. 5 show that the apparent crossing of a collisionopause similar to that observed by RPC-IES, where the ion velocity drops to $\mathrm{km} \mathrm{s}^{-1}$, occurs at a greater distance from the nucleus for comets with higher production rates. During the flyby of Halley, M19/M18 began to rapidly increase inside of this point $\left(r \sim 3 \times 10^{4} \mathrm{~km}\right)$, demonstrating the increasing importance of reaction (R2) with decreasing distance. However, this ratio drops suddenly around $r \sim 1.1 \times 10^{4} \mathrm{~km}$, at the same point where the total ion density drops with decreasing distance, a point suggested to be the ion-pileup boundary (Pätzold et al. 1997; Coates 1997, 2009). No similar feature is evident in Fig. 5 for the other flybys or for the Rosetta excursion. The mechanism proposed for forming an ion-pileup boundary is more efficient loss of ions by electron recombination due to a decrease in electron temperature closer to the nucleus (Ip et al. 1988; Gan \& Cravens 1990; Häberli et al. 1995, 1996; Lindgren et al. 1997). Although the electron temperature in this region could not be measured by Giotto because of spacecraft charging effects (Häberli et al. 1996), we can test this proposed mechanism by comparing the loss rates of the dominant mass 18 ions, $\mathrm{H}_{2} \mathrm{O}^{+}$, and $\mathrm{NH}_{4}^{+}$with that of the dominant mass 19 ion, $\mathrm{H}_{3} \mathrm{O}^{+}$, to see if the drop in M19/M18 can be explained by enhanced electron recombination of $\mathrm{H}_{3} \mathrm{O}^{+}$relative to $\mathrm{H}_{2} \mathrm{O}^{+}$and $\mathrm{NH}_{4}^{+}$. The rate of recombination, $k$, is a function of the electron temperature

$k=a\left(\frac{300}{T_{\mathrm{e}}}\right)^{b}$,

where $a$ and $b$ are constants derived from laboratory measurements or theory, and $T_{\mathrm{e}}$ is the electron temperature. The values for $a$ and $b$ for $\mathrm{H}_{2} \mathrm{O}^{+}, \mathrm{NH}_{4}^{+}$, and $\mathrm{H}_{3} \mathrm{O}^{+}$are given in Table 3. The coefficient $a$ is more than a factor of two larger for $\mathrm{H}_{3} \mathrm{O}^{+}$than for $\mathrm{H}_{2} \mathrm{O}^{+}$so that the loss rate of $\mathrm{H}_{3} \mathrm{O}^{+}$due to electron recombination will be greater than that for $\mathrm{H}_{2} \mathrm{O}^{+}$. However, in this part of the coma, $\mathrm{H}_{2} \mathrm{O}^{+}$loss is dominated by ion-neutral chemistry. The dominant loss process for $\mathrm{NH}_{4}^{+}$, also at mass 18 , is electron recombination. The coefficients, $a$, for $\mathrm{NH}_{4}^{+}$and $\mathrm{H}_{3} \mathrm{O}^{+}$are very close, but the difference in the exponent $b$ means that the loss rate for $\mathrm{H}_{3} \mathrm{O}^{+}$is greater than the loss rate for $\mathrm{NH}_{4}^{+}$at electron temperatures greater than $300 \mathrm{~K}$, or $0.026 \mathrm{eV}$. The electron temperatures in this region of the coma are likely to be higher than this, so that the electron recombination rate of $\mathrm{H}_{3} \mathrm{O}^{+}$will be greater than for $\mathrm{NH}_{4}^{+}$. Therefore, the drop in M19/M18 around the ion-pileup boundary supports the proposal that this boundary is the result of enhanced ion loss that is due to recombination.

It is interesting to note in Fig. 5 that the local neutral densities at Halley were much lower in the apparent collisionopause region than for Rosetta or for 19P/Borrelly, possibly because of photoabsorption in the optically thick coma of Halley (see Beth et al. 2019, Sect. 2.2). In spite of this, Fig. 6 shows that the 
Table 3. Reaction rate coefficients for electron recombination of $\mathrm{H}_{2} \mathrm{O}^{+}$ and $\mathrm{H}_{3} \mathrm{O}^{+}$from Plessis (2010) and for $\mathrm{NH}_{4}^{+}$from Öjekull et al. (2004).

\begin{tabular}{lll}
\hline \hline Ion & $a\left(10^{-7} \mathrm{~cm}^{3} \mathrm{~s}^{-1}\right)$ & $b$ \\
\hline $\mathrm{H}_{2} \mathrm{O}^{+}$ & 3.34 & 0.77 \\
$\mathrm{NH}_{4}^{+}$ & 9.38 & 0.60 \\
$\mathrm{H}_{3} \mathrm{O}^{+}$ & 8.00 & 0.90 \\
\hline
\end{tabular}

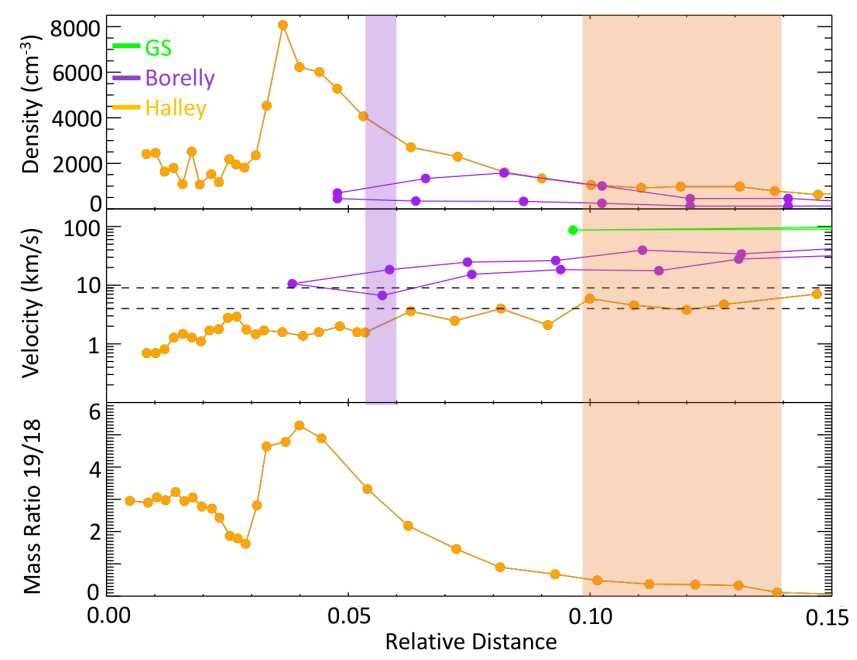

Fig. 7. Plasma observations from the three comet flybys vs. the distance relative to the location of the bow shock. See the caption of Fig. 5 for details.

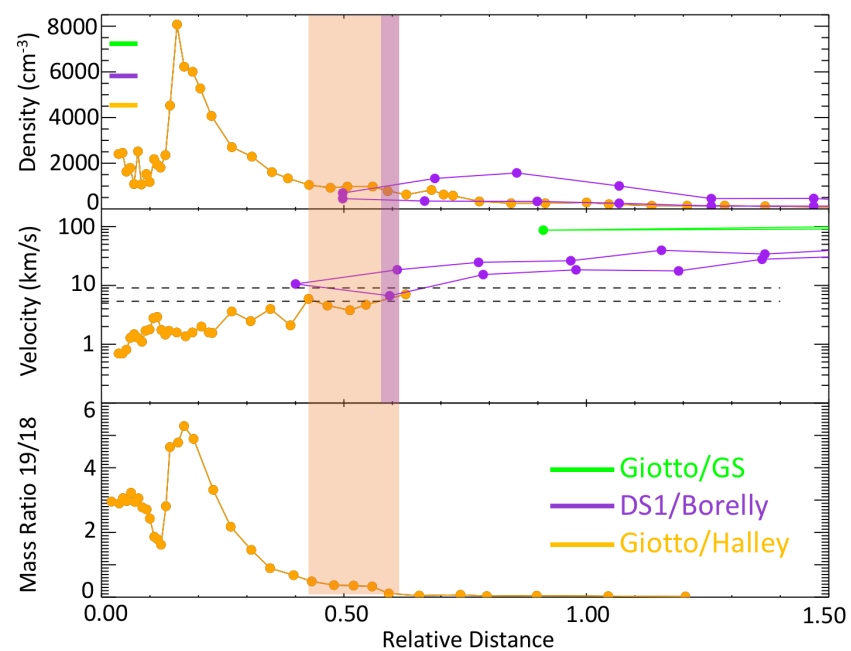

Fig. 8. Plasma observations from the three comet flybys vs. the distance relative to the location of the cometopause. See the caption of Fig. 5 for details.

distance of the observed boundary from the nucleus is proportional to the production rate. The distance for Rosetta covers a wide range, probably because of variability in the solar wind during the time of the excursion, which took place over a period of several weeks.

The location of the apparent collisionopause crossing when the distance is scaled relative to the bow shock is shown in Fig. 7. The location of the bow shock is not related to the collisionopause, which is logical because collisions do not play a role in the formation of the bow shock. However, in Fig. 8, the distance is scaled relative to the cometopause location and there appears to be a relationship between the two. This is reasonable given that the cometopause is thought to be a type of collisionopause

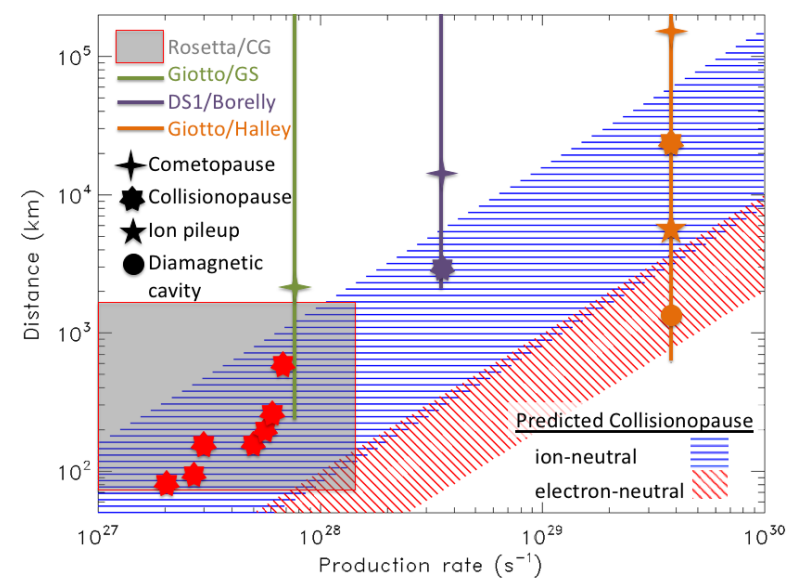

Fig. 9. Comparison of observations of the cometopause, collisionopause, ion-pileup boundary, and diamagnetic cavity boundary for three comet flybys as a function of production rate and distance from the comet. The predicted region for the ion-neutral (blue) and electronneutral (red) collisionopause using Eq. (2) and the cross sections from Mendis et al. (1986) are also shown. Rosetta observations throughout the mission took place in the production and distance range covered by the gray box, and the boundary crossings are from Mandt et al. (2016). The lines for the flybys show the distances covered by the spacecraft.

(see Sect. 2.2). The cometopause was not observed by Rosetta, but based on the observations in the right panel of Fig. 6, it would have been located $830-1750 \mathrm{~km}$ from the nucleus during the excursion. The Giotto flyby of 26P/GS did not approach close enough to the nucleus in terms of relative distance to the cometopause to cross the ion-neutral collisionopause.

In Fig. 9 we compare the location of observed crossings of the cometopause, collisionopause, ion-pileup boundary, and diamagnetic cavity boundary with the production rate for the three flybys, and indicate the area where the ion-neutral and electron-neutral collisionopauses are estimated to be when using the cross sections from Mendis et al. (1986). We chose these cross sections because they were effective at predicting the location of the boundary during the Rosetta excursion (see Sect. 2.2 and Mandt et al. 2016). We include observations from Rosetta over a variety of production rates using observations reported in Mandt et al. (2016) to provide a broader perspective than the limited observations of the excursion.

All of the observed ion-neutral collisionopause crossings are within the predicted region. The ion-pileup boundary crossing observed by Giotto at Halley occurred just outside the area where the electron-neutral collisionopause could be located, providing further support for the proposal that this boundary is formed as a result of cooling of electrons (Ip et al. 1988; Gan \& Cravens 1990; Häberli et al. 1995, 1996; Lindgren et al. 1997; Eriksson et al. 2017; Henri et al. 2017; Engelhardt et al. 2018). The crossing of the diamagnetic cavity boundary observed by Giotto at Halley was well within the location where the electron-neutral collisionopause could be located, and closest approach was clearly inside of this collisionopause. Rosetta observations of the diamagnetic cavity boundary have also been connected to the electron collisionopause (e.g., Mandt et al. 2016; Henri et al. 2017).

\section{Discussion and conclusions}

Based on our evaluation of the predicted location of the ionneutral collisionopause based on cross sections (Fig. 3) and 
our comparison with three other comet flybys (Figs. 5-9), we conclude the following.

- Assuming that the ion velocity is much greater than the neutral velocity (Eq. (2)) provides a reasonable estimate for the location of this collisionopause at ion energies relevant to the RPC-IES observations.

- For a more precise prediction of the collisionopause, in particular, to evaluate solar wind effects, ion velocities need be taken into consideration (Eq. (6)).

- The location of the collisionopause is related to the location of the cometopause (Fig. 8).

- The location of the bow shock is independent of the location of the collisionopause (Fig. 7).

- The location of the collisionopause is directly related to the production rate (Fig. 6), but the location of the ion-neutral collisionopause cannot be predicted by the production rate alone (Fig. 9).

Our study ultimately shows that the ion velocity is required to provide an accurate assessment of where the collisionopause will be located. Rosetta observed a collisionopause at distances greater than $500 \mathrm{~km}$ from the nucleus when the production rate of $67 \mathrm{P}$ was similar to that of $26 \mathrm{P} / \mathrm{GS}$, while Giotto did not observe a collisionopause at closer distances to the nucleus. Unfortunately, there is no simple relationship that describes variability of the ion velocity as a function of solar wind velocity and distance within the coma. Clearly, future efforts are required to understand the collisional processes between ions and neutrals in the inner coma of a comet.

Acknowledgements. Rosetta is an ESA mission with contributions from its Member States and the US National Aeronautics and Space Administration (NASA). K.E.M was supported by NASA through contract 1585002 with the Jet Propulsion Laboratory, California Institute of Technology and through NFDAP 80NSSC18K1233. Work at Imperial College London is supported by STFC of UK under grant ST/N000692/1 and ESA under contract No.4000119035/16/ES/JD. We acknowledge the staff of CDPP and IC for the use of AMDA and the RPC Quicklook database (provided by a collaboration between the Centre de Données de la Physique des Plasmas, supported by CNRS, CNES, Observatoire de Paris and Université Paul Sabatier, Toulouse and Imperial College London, supported by the UK Space Agency). We would like to acknowledge ISSI for the great opportunity it offered us for very valuable discussions on this topic as part of the International Team "Plasma Environment at 67P After Rosetta".

\section{References}

Altwegg, K., Balsiger, H., Geiss, J., et al. 1993, A\&A, 279, 260

Balsiger, H., Altwegg, K., Buhler, F., et al. 1986, Nature, 321, 330

Behar, E., Nilsson, H., Alho, M., Goetz, C., \& Tsurutani, B. 2017, MNRAS, 469, S396

Beth, A., Altwegg, K., Balsiger, H., et al. 2016, MNRAS, 462, S562

Beth, A., Galand, M., \& Heritier, K. L. 2019, A\&A, 630, A47 (Rosetta 2 SI)

Burch, J. L., Goldstein, R., Cravens, T. E., et al. 2007, Space Sci. Rev., 128, 697

Carr, C., Cupido, E., Lee, C. G. Y., et al. 2007, Space Sci. Rev., 128, 629

Coates, A. 1997, Adv. Space Res., 20, 255

Coates, A. J. 2009, AIP Conf. Proc., 1183, 121

Coates, A. J., Johnstone, A. D., \& Neubauer, F. M. 1996, J. Geophys. Res. Space Phys., 101, 27573

Cravens, T. 1989, Adv. Space Res., 9, 293

Cravens, T. E. 1991, Collisional Processes in Cometary Plasmas (Washington, DC: American Geophysical Union), 27

Edberg, N. J. T., Eriksson, A. I., Odelstad, E., et al. 2015, Geophys. Res. Lett., 42, 4263

Engelhardt, I. A. D., Eriksson, A. I., Stenberg Wieser, G., et al. 2018, MNRAS, 477,1296

Eriksson, A. I., Boström, R., Gill, R., et al. 2007, Space Sci. Rev., 128, 729

Eriksson, A. I., Engelhardt, I. A. D., André, M., et al. 2017, A\&A, 605, A15

Fleshman, B. L., Delamere, P. A., Bagenal, F., \& Cassidy, T. 2012, J. Geophys. Res. Planets, 117, 5007
Fuselier, S. A., Shelley, E. G., Balsiger, H., et al. 1988, Geophys. Res. Lett., 15, 549

Galand, M., Héritier, K. L., Odelstad, E., et al. 2016, MNRAS, 462, S331

Galeev, A. A., Gribov, B. E., Gombosi, T., et al. 1986, Geophys. Res. Lett., 13, 841

Gan, L., \& Cravens, T. E. 1990, J. Geophys. Res. Space Phys., 95, 6285

Glassmeier, K.-H., Richter, I., Diedrich, A., et al. 2007, Space Sci. Rev., 128, 649

Goetz, C., Koenders, C., Richter, I., et al. 2016, A\&A, 588, A24

Goetz, C., Volwerk, M., Richter, I., \& Glassmeier, K.-H. 2017, MNRAS, 469, S268

Goldstein, R., Altwegg, K., Fuselier, S. A., et al. 1994, J. Geophys. Res. Space Phys., 99, 19255

Goldstein, R., Burch, J. L., Mokashi, P., et al. 2017, MNRAS, 469, S262

Gombosi, T. I. 1987, Geophys. Res. Lett., 14, 1174

Gringauz, K. I., \& Verigin, M. I. 1991, Permanent and Nonstationary Plasma Phenomena in Comet Halley's Head (Washington, DC: American Geophysical Union), 107

Gringauz, K. I., Gombosi, T. I., Tátrallyay, M., et al. 1986a, Geophys. Res. Lett., 13,613

Gringauz, K. I., Verigin, M. I., Richter, A. K., et al. 1986b, in ESLAB Symposium on the Exploration of Halley's Comet, eds. B. Battrick, E. J. Rolfe, \& R. Reinhard, ESA SP, 250

Gunell, H., Goetz, C., Simon Wedlund, C., et al. 2018, A\&A, 619, L2

Hansen, K. C., Altwegg, K., Berthelier, J.-J., et al. 2016, MNRAS, 462, S491

Henri, P., Vallières, X., Hajra, R., et al. 2017, MNRAS, 469, S372

Heritier, K. L., Henri, P., Vallières, X., et al. 2017, MNRAS, 469, S118

Heritier, K. L., Galand, M., Henri, P., et al. 2018, A\&A, 618, A77

Häberli, R. M., Altwegg, K., Balsiger, H., \& Geiss, J. 1995, A\&A, 297, 881

Häberli, R. M., Altwegg, K., Balsiger, H., \& Geiss, J. 1996, J. Geophys. Res. Space Phys., 101, 15579

Ip, W.-H., Schwenn, R., Rosenbauer, H., et al. 1988, in Exploration of Halley's Comet, eds. M. Grewing, F. Praderie, \& R. Reinhard (Berlin, Heidelberg: Springer), 132

Itikawa, Y., \& Mason, N. 2005, J. Phys. Chem. Ref. Data, 34, 1

Johnstone, A. D., Coates, A. J., Huddleston, D. E., et al. 1993, A\&A, 273, L1

Johnson, R. E., Combi, M. R., Fox, J. L., et al. 2008, Space Sci. Rev., 139, 355

Lindgren, C. J., Cravens, T. E., \& Ledvina, S. A. 1997, J. Geophys. Res. Space Phys., 102, 17395

Lishawa, C. R., Dressler, R. A., Gardner, J. A., Salter, R. H., \& Murad, E. 1990 J. Chem. Phys., 93, 3196

Mandt, K. E., Eriksson, A., Edberg, N. J. T., et al. 2016, MNRAS, 462, S9

Mendis, D. A., Smith, E. J., Tsurutani, B. T., et al. 1986, Geophys. Res. Lett., 13, 239

Mendis, D. A., Flammer, K. R., Rème, H., et al. 1989, Ann. Geophys., 7, 99

Nemeth, Z., Burch, J., Goetz, C., et al. 2016, MNRAS, 462, S415

Neubauer, F. M. 1988, in Exploration of Halley's Comet, eds. M. Grewing, F. Praderie, \& R. Reinhard (Berlin, Heidelberg: Springer), 73

Neubauer, F. M., Glassmeier, K. H., Pohl, M., et al. 1986, Nature, 321, 352

Nilsson, H., Stenberg-Wieser, G., Behar, E., et al. 2017, MNRAS, 469, S252

Odelstad, E., Eriksson, A. I., Edberg, N. J. T., et al. 2015, Geophys. Res. Lett., $42,10,126$

Odelstad, E., Stenberg-Wieser, G., Wieser, M., et al. 2017, MNRAS, 469, S568

Odelstad, E., Eriksson, A. I., Johansson, F. L., et al. 2018, J. Geophys. Res. Space Phys, 123, 5870

Pätzold, M., Neubauer, F. M., Andreev, V. E., \& Gavrik, A. L. 1997, J. Geophy. Res. Space Phys., 102, 2213

Plessis, S. 2010, Ph.D. Thesis, Université Paris-Sud XI, Orsay, France

Rème, H., Sauvaud, J. A., d'Uston, C., et al. 1988, in Exploration of Halley's Comet, eds. M. Grewing, F. Praderie, \& R. Reinhard (Berlin, Heidelberg: Springer), 33

Schwenn, R., Ip, W. H., Rosenbauer, H., et al. 1988, in Exploration of Halley's Comet, eds. M. Grewing, F. Praderie, \& R. Reinhard (Berlin, Heidelberg: Springer), 160

Taylor, M. G. G. T., Altobelli, N., Buratti, B. J., \& Choukroun, M. 2017, Phil. Trans. R. Soc. London, Ser. A Math. Phys. Eng. Sci., 375, 20160262

Timar, A., Nemeth, Z., Szego, K., et al. 2017, MNRAS, 469, S723

Vaisberg, O. L., Smirnov, V. N., Gorn, L. S., \& Iovlev, M. V. 1987, Kosmicheskie Issledovaniia, 25, 867

Vigren, E., Galand, M., Eriksson, A. I., et al. 2015, ApJ, 812, 54

Vigren, E., Altwegg, K., Edberg, N. J. T., et al. 2016, AJ, 152, 59

Vigren, E., André, M., Edberg, N. J. T., et al. 2017, MNRAS, 469, S142

Young, D., Crary, F., Nordholt, J., et al. 2004, Icarus, 167, 80

Öjekull, J., Andersson, P. U., Någård, M. B., et al. 2004, J. Chem. Phys., 120, 7391 